\title{
Generation and attosecond shaping of high coherence free-electron beams for ultrafast TEM
}

\author{
Armin Feist ${ }^{1, *}$, Katharina E. Priebe ${ }^{1}$, Christopher Rathje ${ }^{1,2}$, Nora Bach $^{1}$, Nara Rubiano da \\ Silva $^{1}$, Thomas Danz ${ }^{1}$, Marcel Möller ${ }^{1}$, Till Domröse ${ }^{1}$, Thomas Rittmann ${ }^{1}$, Sergey. . \\ Yalunin $^{1}$, Thorsten Hohage ${ }^{3}$, Murat Sivis ${ }^{1}$, Sascha Schäfer $^{1,2}$, and Claus Ropers ${ }^{1}$ \\ ${ }^{1}$ IV. Physical Institute, University of Göttingen, 37077 Göttingen, Germany \\ ${ }^{2}$ Institute of Physics, University of Oldenburg, 26129 Oldenburg, Germany \\ ${ }^{3}$ Institute for Numerical and Applied Mathematics, \\ University of Göttingen, 37083 Göttingen, Germany
}

\begin{abstract}
We demonstrate the generation and optical control of ultrashort high-coherence electron pulses. The free-electron quantum state is phasemodulated in the longitudinal and transverse dimensions, and the formation of attosecond electron pulse trains is quantitatively probed.
\end{abstract}

\section{Introduction}

Utilizing a stroboscopic approach, ultrafast electron diffraction [1] and microscopy [2, 3] techniques provide for a unique access to nanoscale processes on ultrashort time scales. In a laser-pump/electron-probe scheme, ultrafast transmission electron microscopy (UTEM) combines the nanometer spatial resolution of electron microscopy with the femtosecond temporal resolution of optical spectroscopy. The optical manipulation of the free-electron beam in UTEM in its transverse and longitudinal dimensions promises a significant further enhancement of the technique by facilitating all-optical electron phase plates [4] and a temporal resolution in the attosecond regime. However, the implementation of such concepts requires electron pulses of superior coherence properties.

Here, we describe the implementation of an advanced UTEM instrument utilizing lasertriggered field emitters and discuss its application in the active manipulation of freeelectron quantum states. We prepare tailored longitudinal and transverse momentum superposition states, and demonstrate the preparation and quantitative characterization of attosecond electron pulse trains.

\section{Experiment \& results}

The Göttingen UTEM instrument is based on a JEOL 2100F Schottky field emission TEM, which we modified to allow for optical sample excitation and the generation of ultrashort electron pulses (Fig. 1a) [5,6]. The nanoscopic photoelectron source [5-7] employs localized single-photon photoemission, yielding highly coherent ultrashort electron pulses

*Corresponding author: armin.feist@uni-goettingen.de 


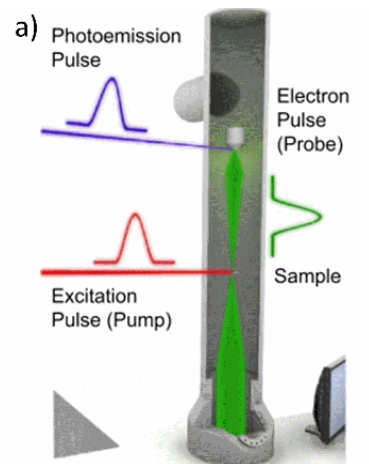

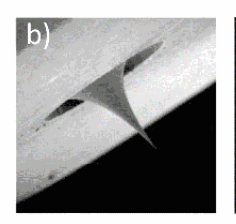
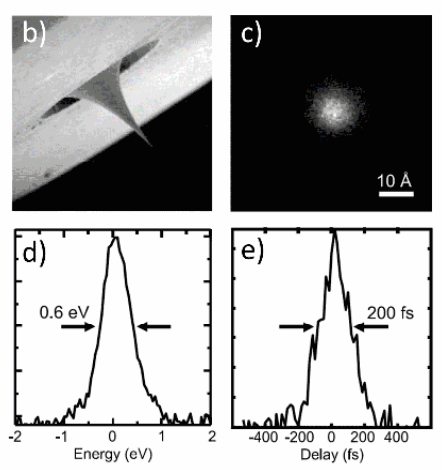

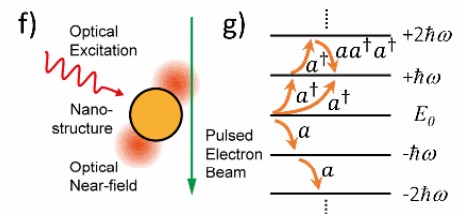

h)

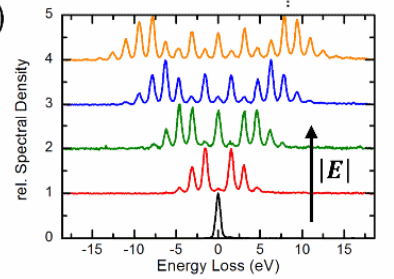

Fig. 1. a) Experimental scheme: Ultrashort electron pulses, generated by nanotip photoemission (b), are accelerated and focused onto a sample (beam properties: c-e). f) The electron beam interacts with an intense optical near field, (h) with its kinetic energy distribution evolving into a comb with multiple spectral sidebands spaced by the photon energy. $g$ ) Energy level diagram of ladder states with spacing $\hbar \omega$ coupled to the initial state at $E_{0}$ (arrows indicate sequential multistate population transfer and interfering quantum paths). Figures adapted from Refs. [5, 6].

with a normalized emittance of a few nm.mrad. Electron focal spot sizes down to $0.8 \mathrm{~nm}$, an electron pulse width of 200 fs (full-width-at-half-maximum) and a spectral bandwidth of $0.6 \mathrm{eV}$ are demonstrated (Fig. 1c-e) [6].

The excellent coherence properties of the pulsed electron beam allow for investigating the optical manipulation of free-electron quantum states [5] by inelastic scattering in localized electromagnetic fields (cf. Fig. 1f) [5, 8-10]. In this interaction, the light field imprints a sinusoidal phase modulation onto the electron wavefunction, corresponding to the absorption and emission of an integer number of photons (cf. Fig. 1g), enabled by the broadened momentum spectrum of confined light fields. The interaction is manifest in a comb of spectral sidebands in the electron kinetic energy distribution (cf. Fig. 1h). The quantum coherent nature of this interaction is evidenced by the observation of Rabi oscillations in the associated momentum state populations [5], and by coherent interactions with multiple, spatially separated light fields in a Ramsey-type geometry [11].

An initial phase-modulated electron state is prepared by overlapping an intense optical field with the pulsed electron beam at a single-crystalline graphite flake (Fig. 2a). Dispersive propagation of the electron wavefunction induces a nearly linear shearing of the quantum state in phase space. As a result, the longitudinal electron density is selfcompressed, evolving into a train of attosecond peaks (Fig. 2b) [5].

A second, spatially separated interaction at a controllable relative phase delay probes the reshaped quantum state by optical coupling of the individual momentum components, imprinting a characteristic kinetic energy distribution onto the electron beam. We retrieve the temporal structure of the electron density distribution (Fig. 2c) by implementing a quantum state tomography technique designed for free-electron beams, termed 'SQUIRRELS' ('spectral quantum interference for the regularized reconstruction of freeelectron states') [12]. Using this technique, we experimentally demonstrate the temporal focusing of the electron beam into a train of attosecond pulses with a duration of about 655 as (FWHM, Fig. 2d).

'SQUIRRELS' is broadly applicable for the analysis of free-electron quantum states, e.g. for multi-color coherent control schemes, constituting an essential building block for free-electron quantum optics. 

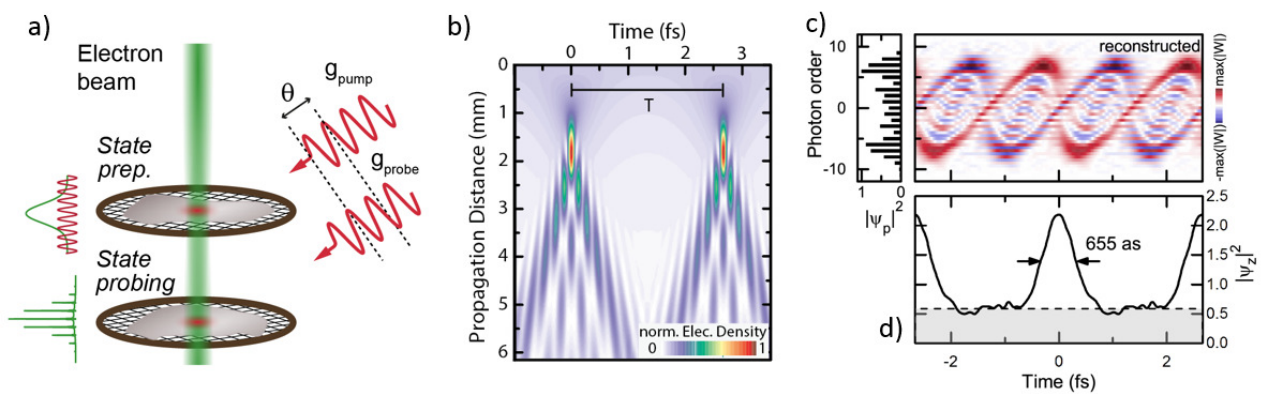

Fig. 2. a) Sketch of the experimental setup employing two graphite flakes for the preparation and characterization of attosecond electron pulse trains. b) Temporal evolution of the electron density after sinusoidal phase modulation as a function of the propagation distance after near-field interaction (numerical simulation for $|g|=5.7$ ). c) Reconstructed Wigner function from experimental data (for $g_{\text {probe }}=3.52$ ) revealing a pronounced shearing due to free-space propagation. d) The temporal projection of the Wigner function exhibits density modulations with a FWHM of 655 as. Figures adapted from Ref. [5, 12].

Finally, we will present the observation of quantized transverse momentum transfer, resulting from momentum conservation in inelastic electron-light scattering at a planar geometry.

\section{Conclusion}

In conclusion, we demonstrated the generation of high brightness, ultrashort electron pulses, and their spatial and temporal shaping by coherent interaction with intense light fields. The ability to tailor and quantitatively map electron quantum states will promote the development of new forms of ultrafast electron microscopy, diffraction and spectroscopy down to the attosecond regime. We envisage the programmable shaping of free-electron wave packets as a basic element of future free-electron quantum optics technology.

\section{References}

1. R.J.D. Miller, Science 343, 1108 (2014)

2. A.H. Zewail, Science 328, 187 (2010)

3. J.S. Kim et al., Science 321, 1472 (2008)

4. H. Müller et al., New J. Phys. 12, 73011 (2010)

5. A. Feist et al., Nature 521, 200 (2015)

6. A. Feist et al., Ultramicroscopy 176, 63 (2017)

7. M. Gulde et al., Science 345, 200 (2014)

8. B. Barwick, D.J. Flannigan, and A.H. Zewail, Nature 462, 902 (2009)

9. F.J. García de Abajo, A. Asenjo-Garcia, and M. Kociak, Nano Lett. 10, 1859 (2010)

10. S.T. Park, M. Lin, and A.H. Zewail, New J. Phys. 12, 123028 (2010)

11. K.E. Echternkamp, A. Feist, S. Schäfer, and C. Ropers, Nat. Phys. 12, 1000 (2016)

12. K.E. Priebe et al., Nat. Photonics 11, 793 (2017) 